\title{
EXTREME POINTS \\ IN THE HAHN-BANACH-KANTOROVIČ SETTING
}

\author{
HaRald LuschgY aNd Wolfgang ThOMSEN
}

\begin{abstract}
This paper presents an existence and characterization theorem for the extreme points of the convex set of all extensions of a linear operator from a real vector space into an order complete real vector lattice which are dominated by a sublinear operator. This result is applied to positive extensions, contractions, and dominated invariant extensions.
\end{abstract}

The paper falls into four sections.

Section 1 is reserved for preliminaries.

In $\$ 2$ we consider the convex set of all extensions of a linear operator defined on a vector subspace of a real vector space $X$ with values in an order complete real vector lattice $Y$ which are dominated by a sublinear operator $P$ from $X$ into $Y$. We present a characterization of the extreme points of this set being useful for applications. This part is related to papers of Kutateladze [7], [8] and Portenier [15].

In $\S 3$ we give two applications of the preceding result. The first one yields another proof of an existence and characterization theorem due to Lipecki [10], [11] concerning extreme positive extensions of a linear operator which is defined on a subspace of an ordered vector space. The second one yields a new characterization theorem for extreme contractions from a separable Banach space into the space of real valued continuous functions on a compact extremally disconnected space.

In $\$ 4$ the results of $\$ 2$ are extended to $P$-dominated extensions which are positive on a given cone in $X$, and we apply them to $P$-dominated extensions which are invariant with respect to a set of mappings from $X$ into $X$. Furthermore, we obtain a refinement of a dominated extension theorem for positive linear operators due to Luxemburg and Zaanen.

1. Preliminaries. We adhere to the notation of Schaefer's monograph [16]. Throughout $X$ stands for a real vector space, $M$ for its vector subspace and $Y$ for an order complete real vector lattice. $P: X \rightarrow Y$ denotes a sublinear mapping, i.e. $P$ is positively homogeneous and subadditive. The space of all linear operators from $M$ into $Y$ is denoted by $L(M, Y)$. Given a vector subspace $N$ of $X$ with $M \subset N$ and $T \in L(M, Y)$, we put

$$
E_{N}(P, T)=\{S \in L(N, Y): S \leq P \mid N \text { and } S \mid M=T\} .
$$


The notation $E_{X}(P, T)$ is abbreviated to $E(P, T)$ and $E(P)$ stands for $E_{X}(P, T)$, provided $M=\{0\}$, i.e.

$$
E(P)=\{S \in L(X, Y): S \leq P\} .
$$

Finally, ex $E_{N}(P, T)$ denotes the set of all extreme points of the convex set $E_{N}(P, T)$.

Proposition 1.1. ( Hahn-Banach-Kantorovič Theorem, [6, 2.5.7, 2.5.8]). If $T \in L(M, Y)$ and $T \leq P \mid M$, then $E(P, T) \neq \varnothing$. In particular, given $x \in X$ and $y \in[-P(-x), P(x)]$, then there exists an operator $S \in E(P)$ such that $S x=y$.

Bonnice, Silverman [3] and To [21] (cf. also Ioffe [5]) have proved that a preordered vector space is order complete, if it has the Hahn-Banach extension property according to Proposition 1.1. Thus the order completeness of $Y$ is indispensable in our investigation of extreme extensions.

2. Existence and characterization of extreme extensions. With $P$ : $X \rightarrow Y$ sublinear and $S \in L(X, Y)$ (and the linear subspace $M$ ) we associate the map $P^{S}: X \rightarrow Y \cup\{-\infty\}$ defined by

$$
\begin{aligned}
P^{S}(x)=\inf \{( & P-S)(u+z+x) \\
& +(P-S)(u-z-x): z \in M, u \in X\} .
\end{aligned}
$$

LEMMA 2.1. The following conditions are equivalent.

(i) $S \leq P$,

(ii) $0 \leq P^{S} \leq 2(P-S)$,

(iii) $P^{S} \mid M=0$,

(iv) $P^{S}(0)>-\infty$,

(v) $P^{S}: X \rightarrow Y$ is sublinear.

The simple proof is left to the reader. (The fact that matters subsequently is that (i) implies the other statements.) Moreover, if $S \leq P$ and $S|M=P| M$, then the definition of $P^{S}$ reduces to

$$
P^{S}(x)=\inf \{(P-S)(u+x)+(P-S)(u-x): u \in X\}
$$

for all $x \in X$.

If $T \in L(M, Y)$ and $T \leq P \mid M$, then the existence of extreme points of $E(P, T)$ is known, see the sophisticated result of Vincent-Smith [22, Addendum to Theorem 1] and recent results of Kutateladze [7], [8] and Lipecki [11]. We shall give a proof using the characterization of extreme extensions stated in part (b) of the following theorem. This characterization turns out to be useful for applications. For $Y=\mathbf{R}$ and 
$M=\{0\}$ it follows from Proposition 2.2 in Portenier [15] and for $M=\{0\}$ some other characterizations may be found in [7], [8].

THEOREM 2.2. Let $T \in L(M, Y)$.

(a) If (and only if ) $T \leq P \mid M$, then ex $E(P, T) \neq \varnothing$.

(b) Suppose $S \in E(P, T)$. Then $S \in$ ex $E(P, T)$ if and only if $P^{S}(x)$ $=0$ for each $x \in X$.

Proof. Both parts (a) and (b) are proved simultaneously. In Step 1 and Step 2 we show the existence of $S \in E(P, T)$ such that $P^{S}=0$ by means of the Kuratowski-Zorn lemma; Step 3 proves the "if" part and Step 4 proves the "only if" part of (b) which completes the proof.

Step 1. By $\mathbf{M}$ we denote the class of all pairs $(N, R)$, where $N$ is a vector subspace of $X$ with $M \subset N$ and $R$ is in $L(N, Y)$ such that $R|M=T, R \leq P| N$ and $P^{R}=0\left(P^{R}(x):=\inf \{(P-R)(u+z+x)\right.$ $+(P-R)(u-z-x): z \in M, u \in N\}, x \in N)$. Let $\ll$ be an ordering in $\mathbf{M}$ defined by $\left(N_{1}, R_{1}\right) \ll\left(N_{2}, R_{2}\right)$ if and only if $N_{1} \subset N_{2}$ and $R_{2} \mid N_{1}$ $=R_{1}$. Given $(N, R) \in \mathbf{M}$ and $x_{0} \in X$, we shall show that $\left(N_{0}, R_{0}\right) \in \mathbf{M}$ and $(N, R) \ll\left(N_{0}, R_{0}\right)$, where $N_{0}=\operatorname{lin}\left(N \cup\left\{x_{0}\right\}\right)$ and $R_{0}: N_{0} \rightarrow Y$ is defined by

$$
R_{0}\left(v+t x_{0}\right)=R v+t y_{0}
$$

$(v \in N, t \in \mathbf{R})$ with

$$
y_{0}=\inf \left\{P\left(v+x_{0}\right)-R v: v \in N\right\} .
$$

As easily seen we have $R_{0} \in L\left(N_{0}, Y\right)$ and $R_{0} \mid N=R$ and $R_{0} \leq P \mid N_{0}$. It remains to show that $P^{R_{0}}=0$. In view of $0 \leq P^{R_{0}} \mid N \leq P^{R}=0$ we get

$$
\begin{aligned}
0 & \leq P^{R_{0}}\left(v+t x_{0}\right) \leq P^{R_{0}}(v)+P^{R_{0}}\left(t x_{0}\right)=|t| P^{R_{0}}\left(x_{0}\right) \\
& =|t| P^{R_{0}}\left(w+x_{0}\right) \leq 2|t|\left(P-R_{0}\right)\left(w+x_{0}\right)
\end{aligned}
$$

for all $v, w \in N$ and $t \in \mathbf{R}$. This yields $P^{R_{0}}=0$, since

$$
\inf \left\{\left(P-R_{0}\right)\left(v+x_{0}\right): v \in N\right\}=0
$$

by the definition of $y_{0}$.

Step 2. Let $\mathbf{M}_{0} \subset \mathbf{M}$ be a chain and put $N_{0}=\bigcup\left\{N:(N, R) \in \mathbf{M}_{0}\right\}$ and $R_{0} \mid N=R$ for all $(N, R) \in \mathbf{M}_{0}$. Then $\left(N_{0}, R_{0}\right)$ in $\mathbf{M}$ is an upper bound for $\mathbf{M}_{0}$, since $0 \leq P^{R_{0}}(x) \leq P^{R}(x)$ for all $x \in N$ and $(N, R) \in$ $\mathbf{M}_{0}$. Thus, by the Kuratowski-Zorn lemma, $\mathbf{M}$ has a maximal element $(N, R)$ and we obtain $N=X$ by Step 1 . 
Step 3. Let $S \in E(P, T)$ with $P^{S}=0$ and $S_{0} \in L(X, Y)$ such that $S \pm S_{0} \in E(P, T)$. Then $S_{0} \mid M=0$ and $\pm S_{0} \leq P-S$. Hence, for each $u, x \in X$ and $z \in M$ we have

$$
\begin{aligned}
2 S_{0} x & =S_{0}(u+z+x)-S_{0}(u-z-x) \\
& \leq(P-S)(u+z+x)+(P-S)(u-z-x)
\end{aligned}
$$

which implies $2 S_{0} \leq P^{S}=0$. Therefore, $S_{0}=0$ whence $S \in \operatorname{ex} E(P, T)$.

Step 4. Given $S \in$ ex $E(P, T)$ and $x_{0} \in X$, there exists $S_{0} \in L(X, Y)$ such that $S_{0}\left(x_{0}\right)=P^{S}\left(x_{0}\right)$ and $S_{0} \leq P^{S}$ by Proposition 1.1. Thus,

$$
\pm S_{0} x=S_{0}( \pm x) \leq P^{S}( \pm x)=P^{S}(x) \leq 2(P-S)(x)
$$

for all $x \in X$ which implies $S \pm \frac{1}{2} S_{0} \leq P$. Moreover, $\pm S_{0} z \leq P^{S}(z)=0$ for all $z \in M$ whence $S \pm \frac{1}{2} S_{0} \in E(P, T)$. Therefore, $P^{S}\left(x_{0}\right)=0$.

The proof of Theorem 2.2 suggests to associate with $P: X \rightarrow Y$ sublinear and $T \in L(M, Y)$ the map $P_{T}: X \rightarrow Y \cup\{-\infty\}$ defined by

$$
P_{T}(x)=\inf \{P(x+z)-T z: z \in M\} \text {. }
$$

Lemma 2.3. Suppose $T \in L(M, Y)$.

(a) The following conditions are equivalent.

(i) $T \leq P \mid M$,

(ii) $T=P_{T} \mid M$,

(iii) $P_{T}(0)>-\infty$,

(iv) $P_{T}: X \rightarrow Y$ is sublinear.

(b) Suppose $S \in L(X, Y)$. Then $S \in E(P, T)$ if and only if $S \leq P_{T}$.

The simple proof is left to the reader. It is worth mentioning that $P^{S}$ and $\left(P_{T}\right)^{S}$ coincide for each $S \in E(P, T)$. Especially, this implies

$$
P^{S}(x)=\inf \left\{\left(P_{T}-S\right)(u+x)+\left(P_{T}-S\right)(u-x): u \in X\right\}
$$

for all $x \in X$. Moreover, if $T \in L(M, Y)$ and $T \leq P \mid M$, then $E(P, T)=$ $E\left(P_{T}\right)$ holds by Lemma 2.3 and according to Proposition 1.1 we obtain

$$
\{S x: S \in E(P, T)\}=\left[-P_{T}(-x), P_{T}(x)\right]
$$

for all $x \in X$. Following up these ideas, we obtain

Corollary 2.4. Suppose $T \in L(M, Y)$ with $T \leq P \mid M$ and $x \in X$. Then

$$
\operatorname{ex}\left[-P_{T}(-x), P_{T}(x)\right] \subset\{S x: S \in \operatorname{ex} E(P, T)\} \subset\left[-P_{T}(-x), P_{T}(x)\right] .
$$


Proof. We only have to show the first inclusion. Given $y \in$ $\operatorname{ex}\left[-P_{T}(-x), P_{T}(x)\right]$, we define

$$
H=\{S \in E(P, T): S x=y\}
$$

and

$$
Q(u)=\sup \{S u: S \in H\}, \quad u \in X .
$$

Then $H \neq \varnothing, Q: X \rightarrow Y$ is sublinear, and $E(Q)=H$. By Theorem 2.2, ex $H \neq \varnothing$. Moreover, $H$ is an extreme subset of $E(P, T)$ by virtue of $y \in \operatorname{ex}\left[-P_{T}(-x), P_{T}(x)\right]$. Thus ex $H \subset \operatorname{ex} E(P, T)$ which implies the assertion.

REMARK 2.5. Both inclusions are proper in general. They provide precise bounds for $\{S x: S \in \operatorname{ex} E(P, T)\}$ as will be shown by the following examples. For this let $(\Omega, \mathcal{Q}, \mu)$ be a probability space. We put $X=L_{1}(\mu), Y=\mathbf{R}, P(u)=\int|u| d \mu$ and $x=1_{\Omega}$. Then

$$
E(P)=\left\{f \in L_{\infty}(\mu):|f| \leq 1_{\Omega}\right\}
$$

and

$$
\operatorname{ex} E(P)=\left\{f \in L_{\infty}(\mu):|f|=1_{\Omega}\right\},
$$

and we have $P(x)=1$ and $-P(-x)=-1$.

If $\mu$ is the one-point measure $\delta_{\omega}$ in $\omega \in \Omega$, then $\left\{\int f d \mu: f \in \operatorname{ex} E(P)\right\}$ $=\{-1,1\} \underset{f}{\subset}[-1,1]$ which shows that the first inclusion turns into equality and the second inclusion is proper.

If $\mu$ is non-atomic, then $\left\{\int f d \mu: f \in \operatorname{ex} E(P)\right\}=[-1,1]$. Indeed, given $|y| \leq 1$, let $y_{0}=(1 / 2)(|y|+1) \in[0,1]$ and $A \in \mathbb{Q}$ with $\mu(A)=y_{0}$. Then $f=1_{A}-1_{A^{c}}$ is in ex $E(P)$ with $\int f d \mu=|y|$. Thus the first inclusion is proper and the second inclusion turns into equality.

REMARK 2.6. Obviously

$$
\left\{-P_{T}(-x), P_{T}(x)\right\} \subset \operatorname{ex}\left[-P_{T}(-x), P_{T}(x)\right]
$$

and this inclusion is proper in general. Indeed, let $(\Omega, \mathcal{Q}, \mu)$ be a probability space, where $\mu$ is not $\{0,1\}$-valued, let $X=\mathbf{R}, Y=L_{\infty}(\mu), P(u)=$ $|u| \cdot 1_{\Omega}$ for all $u \in X$ and $x=1$. Then $\{-P(-x), P(x)\}=\left\{-1_{\Omega}, 1_{\Omega}\right\} \underset{\neq}{\subsetneq}$ $\operatorname{ex}\left[-1_{\Omega}, 1_{\Omega}\right]=\left\{f \in L_{\infty}(\mu):|f|=1_{\Omega}\right\}$.

Suppose now that $M, N$ are linear subspaces of $X$ with $M \subset N \subset X$ and $R \in E_{N}(P, T)$. Theorem 2.7 and the succeeding counterexamples clear up the connections between the sets ex $E_{N}(P, T)$, ex $E(P, T)$ and 
ex $E(P, R)$. In particular, it follows that $S \in \operatorname{ex} E(P, T)$ implies $S \in$ ex $E(P, S \mid N)$, whereas $S \mid N \notin \operatorname{ex} E_{N}(P, T)$ in general. Conversely, $S \in$ ex $E(P, S \mid N)$ and $S \mid N \in \operatorname{ex} E_{N}(P, T)$ imply $S \in \operatorname{ex} E(P, T)$.

THEOREM 2.7. Let $M, N$ be subspaces of $X$ with $M \subset N \subset X$. Suppose $T \in L(M, Y)$ and $R \in E_{N}(P, T)$. Then

(a) ex $E(P, R) \supset E(P, R) \cap \operatorname{ex} E(P, T)$.

(b) Suppose $R \in$ ex $E_{N}(P, T)$. Then

$$
\operatorname{ex} E(P, R)=E(P, R) \cap \operatorname{ex} E(P, T) .
$$

Proof. (a) is obvious and as regards (b), $R \in$ ex $E_{N}(P, T)$ implies that $E(P, R)$ is an extreme subset of $E(P, T)$ which proves the assertion.

REMARK 2.8. The inclusion in (a) is proper in general. Indeed, let $N=X=Y=\mathbf{R}, M=\{0\}, P(x)=|x|$ and $R x=0$ for all $x \in \mathbf{R}$. Then $\{R\}=E(P, R)=\operatorname{ex} E(P, R)$ and ex $E(P)=\left\{\mathrm{id}_{\mathbf{R}},-\mathrm{id}_{\mathbf{R}}\right\}$.

The converse in (b) does not hold as the following example shows (cf. also Singer [19, p. 106]). Let $X=\mathbf{R}^{2}, Y=\mathbf{R}, M=\{(0,0)\}, N=\mathbf{R} \times\{0\}$, $R x=0$ for all $x \in N$ and let $P\left(\left(x_{1}, x_{2}\right)\right)=\max \left\{\left|x_{1}\right|,\left|x_{2}\right|\right\}$ and $\operatorname{pr}_{i}\left(\left(x_{1}, x_{2}\right)\right)=x_{i}$ for all $\left(x_{1}, x_{2}\right) \in \mathbf{R}^{2}$. Then ex $E(P, R)=\left\{\mathrm{pr}_{2},-\mathrm{pr}_{2}\right\} \subset$ $\operatorname{ex} E(P)=\left\{\operatorname{pr}_{1},-\operatorname{pr}_{1}, \mathrm{pr}_{2},-\mathrm{pr}_{2}\right\}$, whereas $R \notin \operatorname{ex} E_{N}(P)=\left\{\operatorname{pr}_{1} \mid N\right.$, $\left.-\operatorname{pr}_{1} \mid N\right\}$.

In particular, this example yields an operator $S \in$ ex $E(P)$ such that $S \mid N \notin \operatorname{ex} E_{N}(P)$; put $S=\mathrm{pr}_{2}$.

3. Applications. By Theorem 2.2 we obtain immediately a result due to Lipecki concerning extreme positive extensions of an operator defined on a subspace of an ordered vector space with values in an order complete vector lattice.

A1. (Lipecki [10, Theorem 1], [11, Theorem 2 and Remark 2]). Let $X$ be an ordered vector space with positive cone $C, M$ a majorizing vector subspace of $X$ and $T: M \rightarrow Y$ a positive linear operator. Then

(a) $\operatorname{ex}\{S \in L(X, Y): S \mid M=T$ and $S \mid C \geq 0\} \neq \varnothing$.

(b) Suppose $C$ is generating. Then $S \in\left\{S^{\prime} \in L(X, Y): S^{\prime} \mid M=T\right.$, $\left.S^{\prime} \mid C \geq 0\right\}$ is an extreme point of this set if and only if

$$
\inf \{S u: \pm(x+z) \leq u \in X, z \in M\}=0
$$

for each $x \in X$.

Proof. Obviously, $P: X \rightarrow Y$ defined by

$$
P(x)=\inf \{T z: x \leq z \in M\}
$$


is sublinear and we have

$$
E(P, T)=\{S \in L(X, Y): S|M=T, S| C \geq 0\}
$$

Moreover, it is readily verified that

$$
P^{S}(x)=2 \cdot \inf \{S u: \pm(x+z) \leq u \in X, z \in M\}
$$

holds for all $x \in X$ and $S \in E(P, T)$. Thus the assertion follows from Theorem 2.2.

We now apply Theorem 2.2 to prove a new result concerning extreme contractions into spaces of continuous functions. This subject was started by Blumenthal et al. [2].

Let $X$ be a normed vector space and $Y=C(H)$, where $H$ is an extremally disconnected compact space. Recall that a compact space is extremally disconnected (i.e. open subsets have an open closure) if and only if the space of continuous real valued functions is order complete (cf. [16, II. 7.7]). $X^{\prime}$ denotes the continuous dual of $X, U\left(X^{\prime}\right)$ is the unit ball of $X^{\prime}$ and $U(X, C(H))$ is the unit ball of $L(X, C(H))$. An operator $S \in$ $U(X, C(H))$ is called almost nice, if $S^{\prime}: H \rightarrow U\left(X^{\prime}\right)$ defined by $S^{\prime}(h)=$ $\delta_{h} \circ S$ maps a dense subset of $H$ into ex $U\left(X^{\prime}\right)$.

It is immediate from Theorem 2.2 (with $M=\{0\}$ and $P(x)=\|x\|$ ) that $x^{\prime} \in U\left(X^{\prime}\right)$ is an extreme point of this set if and only if

$$
\inf \left\{\|u+x\|+\|u-x\|+2 x^{\prime}(u): u \in X\right\}=0
$$

for each $x \in X$. (Incidentally, this characterization may be used to prove the well known result that ex $U\left(C^{\prime}(K)\right)=\left\{\alpha \delta_{k}:|\alpha|=1, k \in K\right\}$, where $K$ is a compact space [4, V.8.6]). Furthermore, let us point out that an almost nice operator $S$ in $U(X, C(H))$ is an extreme point of this set, since $S^{\prime}$ is weak* continuous [4, VI.7.1]. The converse does not hold in general (cf. Remark 3.1). In addition, we note (cf. Oates [14, Theorem 1.2]) that $U(X, C(H))$ is the closed convex hull of its extreme points with respect to the strong (or equivalently weak) operator topology on the space of all continuous linear operators from $X$ into $C(H)$. More general Krein-Milman type theorems may be found in Morris and Phelps [13] and (without proofs) in Levashov [9].

A2. Let $X$ be a separable normed space and let $H$ be an extremally disconnected compact space. Then $S \in U(X, C(H))$ is an extreme point of this set if and only if $S$ is almost nice. 
Proof. We only have to show the "only if" part. Suppose $S \in$ ex $U\left(X, C(H)\right.$ ). By Theorem 2.2 (with $M=\{0\}$ and $P(x)=\|x\| 1_{H}$ ), we have

$$
\inf \left\{\|u+x\| 1_{H}+\|u-x\| 1_{H}-2 S u: u \in X\right\}=0
$$

for each $x \in X$. Let $\varphi: X \times H \rightarrow \mathbf{R}$ be defined by

$$
\varphi(x, h)=\inf \left\{\|u+x\|+\|u-x\|-2 \delta_{h}(S u): u \in X\right\} .
$$

The set $\{\varphi(x, \cdot)>0\} \subset H$ is meager for each $x \in X$, since the lattice infimum and the point infimum in $C(H)$ differ on a meager subset (cf. Stone [20]). If $A$ is a countable dense subset of $X$, then

$$
K=\bigcap_{x \in A}\{\varphi(x, \cdot)=0\}
$$

is a dense subset of $H$ by Baire's category theorem. Since $\varphi(\cdot, h)$ is continuous for all $h \in H$, we obtain $\varphi(\cdot, h)=0$ for all $h \in K$. Thus by Theorem $2.2 T$ is almost nice.

Without proof we note the following slight generalization of A2.

A3. Let $X$ be a separable normed space, $M$ a vector subspace of $X$ and $T \in U(M, C(H))$, where $H$ is an extremally disconnected compact space. Then

$$
S \in\{R \in U(X, C(H)): R \mid M=T\}
$$

is an extreme point of this set if and only if

$$
S^{\prime}(h) \in \operatorname{ex}\left\{x^{\prime} \in U\left(X^{\prime}\right): x^{\prime} \mid M=T^{\prime}(h)\right\}
$$

for all $h$ in some dense subset of $H$.

Remark 3.1. A2 and A3 fail for non-separable normed spaces $X$. Indeed, let $H$ be a compact extremally disconnected space such that the set $H_{0}$ of isolated points of $H$ is not dense (e.g. if $(\Omega, \mathcal{Q}, \mu)$ is a positive $\sigma$-finite non-atomic measure space and $\Re$ the ideal of $\mu$-null sets, then the Stone representation space of $Q / \mathcal{T}$ is extremally disconnected and has no isolated points [18, p. 28 and p. 86]). By virtue of a result due to Blumenthal et al. [2, Theorem 2] there is a (non-separable) Banach space $X$ and an operator $S \in$ ex $U(X, C(H))$ such that $\left\{h \in H: S^{\prime}(h) \in\right.$ ex $\left.U\left(X^{\prime}\right)\right\}=H_{0}$. Hence $S$ is not almost nice.

Nevertheless, the separability of $X$ can be removed if $X=C(K)$ for some compact space $K$. More generally, the separability assumption in A2 can be replaced by the assumption that ex $U\left(X^{\prime}\right)$ is weak* closed. This result is due to Sharir [17] and it may also be proved by an application of Theorem 2.2 and Theorem 2.7. 
4. Generalizations. In this section the preceding results are generalized to $P$-dominated extensions, which are positive on a (pointed convex) cone $C \subset X$. With $P: X \rightarrow Y$ sublinear and a cone $C \subset X$ we associate the map $P_{C}: X \rightarrow Y \cup\{-\infty\}$ defined by

$$
P_{C}(x)=\inf \{P(x+u): u \in C\}
$$

and, given $T \in L(M, Y)$, we put

$$
E(P, T, C)=\{S \in E(P, T): S \mid C \geq 0\} .
$$

LEMMA 4.1. Let $C$ be a cone in $X$.

(a) The following conditions are equivalent.

(i) $P \mid C \geq 0$,

(ii) $P_{C}(0)>-\infty$,

(iii) $P_{C}: X \rightarrow Y$ is sublinear.

(b) Suppose $S \in L(X, Y)$. Then $S \leq P$ and $S \mid C \geq 0$ if and only if $S \leq P_{C}$.

The simple proof will be omitted (for $Y=\mathbf{R}$ compare Anger and Lembcke [1, Lemma 1.9, Lemma 3.2]). In view of the preceding lemma the following corollary is merely a restatement of Theorem 2.2 .

Corollary 4.2. Let $T \in L(M, Y)$ and $C$ be a cone in $X$.

(a) If (and only if) $T \leq P_{C} \mid M$, then ex $E(P, T, C) \neq \varnothing$.

(b) An operator $S \in E(P, T, C)$ is an extreme point of this set if and only if $\left(P_{C}\right)^{S}(x)=0$ for each $x \in X$.

REMARK 4.3. Lemma 2.3 yields equivalent assertions for the statement $T \leq P_{C} \mid M$. Furthermore, $T \leq P_{C} \mid M$ yields

$$
E(P, T, C)=E\left(P_{C}, T\right)=E\left(P_{T}, C\right)=E\left(P_{T C}\right),
$$

where $P_{T C}$ stands for both (coinciding) operators $\left(P_{C}\right)_{T}$ and $\left(P_{T}\right)_{C}$. Finally, we note that $T \leq P_{C} \mid M$ readily implies $T \leq P|M, T| M \cap C \geq 0$, $P \mid C \geq 0$, but the converse does not hold in general. Indeed, let $X=\mathbf{R}^{2}$, $M=\mathbf{R} \times\{0\}, C=\{0\} \times \mathbf{R}, \quad Y=\mathbf{R}, \quad P\left(\left(x_{1}, x_{2}\right)\right)=\left|x_{1}+x_{2}\right|$ for all $\left(x_{1}, x_{2}\right) \in X$ and $T\left(x_{1}, 0\right)=x_{1}$ for all $\left(x_{1}, 0\right) \in M$ and note that $P_{C} \mid M=$ 0 .

An application of Corollary 4.2 yields a refinement of a result due to Luxemburg and Zaanen.

A4. (Luxemburg and Zaanen; cf. [6, 2.6.3]). Suppose that $X$ is a vector lattice with positive cone $C, M$ is a vector sublattice, and $P$ is lattice-increasing, i.e. $\left|x_{1}\right| \leq\left|x_{2}\right|$ implies $P\left(x_{1}\right) \leq P\left(x_{2}\right), x_{i} \in X$. Let $T \in L(M, Y)$.

(a) If $T \leq P \mid M$ and $T \mid M \cap C \geq 0$, then ex $E(P, T, C) \neq \varnothing$. 
(b) An operator $S \in E(P, T, C)$ is extreme in this set if and only if $\inf \left\{P\left((u+z+x)^{+}\right)+P\left((u-z-x)^{+}\right)-2 S u: z \in M, u \in X\right\}=0$

for each $x \in X$.

Proof. First note that $P_{C}(x)=P\left(x^{+}\right)$holds for all $x \in X$. Indeed, $x \leq x^{+}$implies $P_{C}(x) \leq P\left(x^{+}\right)$; conversely, $x \leq u \in X$ implies $x^{+} \leq u^{+}$, and since $P$ is lattice-increasing we obtain $P\left(x^{+}\right) \leq P\left(u^{+}\right) \leq P(u)$ whence $P\left(x^{+}\right) \leq P_{C}(x)$. Moreover, we have $T z \leq T z^{+} \leq P\left(z^{+}\right)$for all $z \in M$. Hence the assertions follow from Corollary 4.2.

In the remaining part of this section we deal with invariant $P$ dominated extensions. Let $\mathcal{G}$ be a set of mappings from $X$ into $X$. A linear operator $S: X \rightarrow Y$ is called invariant if $S V=S$ for all $V \in \mathcal{G}$. The vector space of all invariant linear operators from $X$ into $Y$ is denoted by $L(X, Y)_{\mathcal{G}}$ and, given $T \in L(M, Y)$, we put

$$
E(P, T)_{\mathcal{G}}=E(P, T) \cap L(X, Y)_{\mathcal{G}} .
$$

Furthermore, let $G$ denote the linear hull of the set $\{V x-x: V \in \mathcal{G}, x \in$ $X$ \}. Obviously, $S \in L(X, Y)$ is invariant if and only if $S \mid G=0$, i.e. $G \subset \operatorname{ker} S$.

A5. Let $T \in L(M, Y)$ and $\mathcal{G}$ be a set of mappings from $X$ into $X$.

(a) If (and only if) $T \leq P_{G} \mid M$, then ex $E(P, T)_{\mathcal{S}} \neq \varnothing$.

(b) $S \in E(P, T)_{\mathcal{G}}$ is an extreme point of this set if and only if

$$
\begin{aligned}
\inf \{(P-S)(u+z+x)+(P-S)(u-z-x): & \\
& (z \in M+G, u \in X\}=0
\end{aligned}
$$

for each $x \in X$.

Proof. Obviously, $E(P, T)_{\mathcal{G}}=E(P, T, G)$, and it is easily seen that $\left(P_{G}\right)^{S}=\left(P^{S}\right)_{G}$ holds for all $S \in E(P, T, G)$. Hence the assertions follow from Corollary 4.2.

A6. Let $T$ and $\mathcal{G}$ be as in A5. Assume that there exists an operator $R \in L(X, X)$ such that $R M \subset M, P R \leq P$ and $P_{\text {ker } R} \leq P_{G}$.

(a) If $T R=T$ on $M$ and $T \leq P \mid M$, then ex $E(P, T)_{\Theta} \neq \varnothing$.

(b) If additionally $R$ is a projection with $P_{\text {ker } R}=P_{G}$, then an operator $S$ in $E(P, T)_{\mathcal{S}}$ is an extreme point of this set if and only if $P^{S}(x)=0$ for each $x$ in the fixed space of $R$.

Proof. (a) For $z \in M$ and $u \in \operatorname{ker} R$ we get $T z=T R z \leq P(R z)=$ $P(R(z+u)) \leq P(z+u)$ whence $T \leq P_{\text {ker } R} \mid M$. The assertion follows from A5. 
(b). Suppose $S \in E(P, T)_{g}$. Then $S \leq P_{G}=P_{\text {ker } R}$ implies $S R=S$ whence $(P R)^{S}=P^{S} R$. Furthermore, it is readily verified that $P R=P_{\mathrm{ker} R}$. Therefore $\left(P_{G}\right)^{S}=P^{S} R$. By virtue of Corollary $4.2 S$ is an extreme point of $E(P, T)_{\mathcal{S}}$ if and only if $P^{S}(R x)=0$ for each $x \in X$, i.e. $P^{S}(x)=0$ for each $x$ in the fixed space of $R$.

We conclude our considerations by applying this result to a topological setting. A similar result for positive invariant extensions was stated by the first-named author in [12].

A7. Suppose that $X$ is a locally convex space, that $\mathcal{G}$ is a mean ergodic semigroup of continuous linear operators on $X[16$, III.7.1] and that the order complete vector lattice $Y$ is a topological vector space with a closed normal positive cone (e.g. we may assume that $Y$ is an order complete topological vector lattice). Let $M$ be a closed subspace and let $P$ be continuous such that $V M \subset M$ and $P V \leq P$ for all $V \in \mathcal{G}$. Suppose $T \in L(M, Y)$.

(a) If $T$ is invariant and $T \leq P \mid M$, then ex $E(P, T)_{\mathcal{S}} \neq \varnothing$.

(b) An operator $S$ in $E(P, T)_{\mathcal{S}}$ is an extreme point of this set if and only if $P^{S}(x)=0$ for each $x$ in the fixed space of $\mathcal{G}$.

Proof. Let $R$ be the zero element of the closed convex hull of $\mathcal{G}$ in the space of all continuous linear operators on $X$ equipped with the topology of pointwise convergence. $R$ is a continuous linear projection onto the fixed space of $\mathcal{G}$ with kernel the closure of $G$ [16, III.7.2]. In view of the properties of $P$ and $\mathcal{G}$ we obtain $P_{\text {ker } R}=P_{G}, P R \leq P$ and $R M \subset M$. Furthermore, each $S \in E(P)$ is continuous since $P$ is continuous and the positive cone of $Y$ is normal. Thus $T$ is continuous by virtue of $E(P, T) \neq$ $\varnothing$ and employing the invariance of $T$ we obtain $T R=T$ on $M$. The assertions follow now from A6.

Remark 4.4. If $\mathcal{G}$ is a set of mappings from $X$ into $X$, then invariant versions of $\mathrm{A} 2$ and $\mathrm{A} 3$ are valid.

Acknowledgement. We are grateful to $\mathrm{Z}$. Lipecki for his careful reading of this paper and his suggestions.

\section{REFERENCES}

1. B. Anger and L. Lembcke, Hahn-Banach type theorems for hypolinear functionals on preordered topological vector spaces, Pacific J. Math., 54 (1974), 13-33.

2. R. M. Blumenthal, J. Lindenstrauß and R. R. Phelps, Extreme operators into $C(K)$, Pacific J. Math., 15 (1965), 747-756.

3. W. E. Bonnice and R. J. Silverman, The Hahn Banach extension and the least upper bound properties are equivalent, Proc. Amer. Math. Soc., 18 (1967), 843-849. 
4. N. Dunford and J. T. Schwartz, Linear Operators, Part 1, Interscience Publishers, New York, 1967.

5. A. D. Ioffe, A new proof of the equivalence of the Hahn-Banach extension and the least upper bound property, Proc. Amer. Math. Soc., 82 (1981), 385-389.

6. G. Jameson, Ordered Linear Spaces, Springer-Verlag, Berlin-Heidelberg-New York, 1970.

7. S. S. Kutateladze, Extreme points of subdifferentials, Soviet. Math. Dokl., 19 (1978), 1235-1237.

$8 . \quad$ The Krein-Mil'man theorem and its inverse, Siberian Math. J., 21 (1980), 97-103.

9. V. A. Levashov, Operator analogs of the Krein-Milman theorem, Functional Anal. Appl., 14 (1980), 130-131.

10. Z. Lipecki, Extensions of positive operators and extreme points II, Colloquium Math., 42 (1979), 285-289.

11. Extensions of positive operators and extreme points III, Colloquium Math., 46 (1982), 263-268.

12. H. Luschgy, Invariant extensions of positive operators and extreme points, Math. Z., 171 (1980), 75-81.

13. P. D. Morris and R. R. Phelps, Theorems of Krein-Milman type for certain convex sets of operators, Trans. Amer. Math. Soc., 150 (1970), 183-200.

14. D. K. Oates, A non-compact Krein-Milman theorem, Pacific J. Math., 36 (1971), 781-785.

15. C. Portenier, Points extrémaux et densité, Math. Ann., 209 (1974), 83-89.

16. H. H. Schaefer, Banach Lattices and Positive Operators, Springer-Verlag, BerlinHeidelberg-New York, 1974.

17. M. Sharir, Characterization and properties of extreme operators into $C(Y)$, Israel J. Math., 12 (1972), 174-183.

18. R. Sikorski, Boolean Algebras, 3rd Edition, Springer-Verlag, Berlin-Göttingen-Heidelberg-New York, 1964.

19. I. Singer, Sur l'extension des fonctionelles linéaires, Revue Math. Pures Appl., 1 (1956), 99-106.

20. M. H. Stone, Boundedness properties in function lattices, Canad. J. Math., 1 (1949), $176-186$.

21. Ting-on To, The equivalence of the least upper bound property and the Hahn-Banach extension property in ordered linear spaces, Proc. Amer. Math. Soc., 30 (1971), 287-295.

22. G. Vincent-Smith, The Hahn-Banach theorem for modules, Proc. London Math. Soc., 17 (1967), 72-90.

Received May 22, 1981 and in revised form March 8, 1982.

STIFTSHERRENSTRASSE 5

D-4400 MUNSTER

FRG

AND

WESTFÄLISCHE WILHELMS-UNIVERSITÄT

EINSTEINSTRASSE 62

D-4400 MÜNSTER

FRG 